\title{
A voz universitária: promoção da saúde e prevenção da Covid-19 via rádio
}

\author{
The university voice: health promotion and prevention of Covid-19 via radio
}

\author{
Ana Karla da Silva Dantas ${ }^{1}$ (D) $\mid$ dantasana161@gmail.com \\ Mirian Lourenço Santos ${ }^{1}$ (D) mirian.santos@altamira.ufpa.com.br \\ Lucas Mendes Carvalho' ${ }^{1}$ (D) lucasmencarvalho@hotmail.com \\ Adrianne Carla de Castro Tomé1 (1) adriannetome@hotmail.com \\ Ramon Veloso Sousa Sobral' (1) ramon.sobral@altamira.ufpa.br \\ Ozélia Sousa Santos ${ }^{1}$ (D) ozeliasousa@hotmail.com
}

\begin{abstract}
RESUMO
Introdução: A necessidade de garantir a autonomia do indivíduo na construção do seu bem-estar evidencia a busca de novas formas de levar conhecimento sobre saúde à população. Uma delas, pelos meios de comunicação em massa, como o rádio, pode alcançar públicos nas mais longínquas localidades, de modo a dinamizar o processo de melhoria da qualidade de vida e mudar o paradigma dos processos de saúde. Nesse sentido, nasce o projeto de extensão “A voz universitária" que leva informações de saúde e bem-estar, pela Rádio 93.1 FM, para mais de 400 mil habitantes na região da Transamazônica. Este trabalho tem como objetivo descrever as experiências dos integrantes desse projeto durante sua primeira fase.

Relato de experiência: O projeto contribuiu com a distribuição de materiais informativos de formas física (cartilhas educativas) e digital e via rádio sobre temáticas gerais de saúde, qualidade de vida e cidadania. Distribuíram-se mais de três mil unidades da cartilha impressa a comunidades ribeirinhas do Xingu, além da disponibilização no formato digital à Secretaria Municipal de Educação de Altamira. Além disso, ao longo dos primeiros seis meses de implementação, o projeto abordou via rádio mais de 40 temáticas e reuniu mais de 20 profissionais de saúde locais para conversar com o público.
\end{abstract}

Discussão: A garantia da autonomia do indivíduo para identificar sua urgência de ajuda e procurar auxílio quando necessário é uma forma de dinamizar as práticas de saúde, com especial atenção às comunidades historicamente marginalizadas e socialmente vulneráveis como os indígenas e ribeirinhos do Xingu, que sem acesso a outros meios de comunicação, senão o rádio, teriam mais dificuldade em receber essas informações. Assim, os meios de comunicação em massa se mostram efetivos na construção de um saber científico inclusivo para comunidades tradicionais.

Conclusão: Os meios de comunicação em massa e comunicação digital são importantes ferramentas para a prática médica, pois permitem que esta saia dos muros do modelo biomédico e curativista e abra novos horizontes para a resolução dos problemas de saúde sem a necessidade da intervenção direta de um profissional, de modo a garantir a autonomia do indivíduo na construção do seu bem-estar e reaproximar a comunidade acadêmica da sociedade.

Palavras-chave: Educação em Saúde; Qualidade de Vida; Autonomia.

\section{ABSTRACT}

Introduction: The need to guarantee the individual's autonomy in the construction of their well-being demonstrates new ways of informing the public about health. One of these, the mass media, like radio, can reach people in the most distant locations, streamlining the process of improving quality of life and changing the paradigm of health processes. Thus, the community outreach project "University Voice" spread health information to more than 400,000 people in the Transamazonica region. This article aims to describe the experiences of the members of this project during the first phase of its implementation.

Experience report: The project contributed to the dissemination of physical informative materials (educational booklets), digital materials and information via radio about health, quality of life and citizenship. Three thousand booklets were distributed. Furthermore, the booklet was provided to the city's Education Department in digital format. For six months, the project addressed more than forty topics and twenty local health workers had the opportunity to speak with to listeners of the radio station.

Discussion: Ensuring the individual's autonomy to identify the need for help and seek it when necessary is a way to enhance health practices, with special attention to historically marginalized and socially vulnerable communities such as the indigenous and riverside inhabitants of Xingu, who, without access to other means of communication but radio, face more difficulty in receiving this information. The mass media, therefore, are effective in building inclusive scientific knowledge in traditional communities and among the general public.

Conclusion: Mass media and digital communication become important tools for medical practice, leaving the walls of the biomedical and curative model and expanding the horizons for health care without the direct intervention of a physician. This can guarantee the individual autonomy in the construction of a healthy life and bring the academic community closer to the public.

Keywords: Health Education; Quality of Life; Autonomy.

${ }^{1}$ Universidade Federal do Pará, Altamira, Pará, Brasil.

Editora-chefe: Rosiane Viana Zuza Diniz.

Editora associada: Ana Cláudia Santos Chazan.

Recebido em 28/04/21; Aceito em 25/10/21.

Avaliado pelo processo de double blind review. 


\section{INTRODUÇÃO}

Segundo a Conferência Internacional sobre Promoção da Saúde, realizada em Ottawa, no Canadá, em 1986, promoção de saúde consiste no processo de capacitação da comunidade para atuar na melhoria da sua qualidade de vida e saúde, incluindo maior participação no controle desse processo. Sua definição tem um sentido mais amplo que a de prevenção, dado que as medidas adotadas não se dirigem a uma determinada doença ou desordem, mas auxiliam na melhoria da saúde e do bem-estar, enfatizando a transformação das condições de vida e trabalho de uma população'.

Considerando que a educação em saúde deve oferecer condições para que as pessoas desenvolvam o senso de responsabilidade tanto para saúde própria quanto para a coletiva, é importante elaborar meios estratégicos de comunicação que proporcionem uma transformação dos indivíduos socialmente inseridos no mundo². Nesse sentido, o rádio configura-se como uma estratégia de comunicação reconhecida internacionalmente por promover o desenvolvimento social e mudanças de comportamento, e por impactar positivamente a saúde dos ouvintes ${ }^{3}$.

Segundo Torres et al. ${ }^{4}$, a tecnologia da informação e comunicação facilita a comunicação e dinamiza o processo do aprendizado. Assim, o uso de ferramentas de comunicação como o rádio constitui uma importante estratégia de disseminação do conhecimento com qualidade, sobretudo para povos tradicionais, em um cenário de distanciamento social, tendo em vista a situação pandêmica provocada pela coronavirus disease 2019 (Covid-19).

De acordo com Hugelius et al. ${ }^{5}$, o rádio pode ser usado para promover aspectos físicos e psicossociais e bem-estar, propiciando a divulgação de informações relacionadas à saúde ${ }^{5}$. $O$ aconselhamento ativo sobre onde procurar serviços de saúde, a divulgação de atividades e serviços disponíveis e a promoção do autocuidado por meios de rádios são ações internacionalmente reconhecidas como capazes de aumentar proativamente a conscientização do público e possibilitar uma maior e melhor utilização dos serviços públicos ofertados, o que se refletirá na redução da mortalidade e morbidade ${ }^{5}$.

Ademais, percebe-se que, dada a extensão territorial do Brasil, podem-se encontrar populações não apenas residentes em áreas urbanas e rurais, mas também às margens de rios, as denominadas comunidades ribeirinhas, as quais estão isoladas não apenas da cultura geral, mas também do acesso às mídias escrita e televisiva e muitas vezes à internet ${ }^{1}$. Nesses lugares, a mídia radiofônica é o principal instrumento de comunicação com o mundo.

Essas comunidades sofrem influência da dinâmica das águas com suas cheias e vazantes, que impõem restrições e dinamizam o dia a dia do povo ribeirinho, e, por essa razão, vivem economicamente dependentes das atividades do extrativismo vegetal e da pesca. Por causa da distância dessas comunidades dos centros urbanos, da falta de recursos financeiros e de transporte, o acesso aos serviços de saúde fica prejudicado. Essas condições habitacionais exercem influência negativa na qualidade de vida dessa população ${ }^{6}$. Assim, programas de rádio focados nessa população têm o poder de promover a inclusão, ao propiciar a ela liberdade de expressão e acesso a informações de qualidade, além de contribuir para o seu empoderamento ${ }^{7}$.

Nesse sentido, percebe-se que a universidade, como instituição geradora de conhecimentos e formação de profissionais, pode atuar de forma conjunta com a rádio local na socialização das informações sobre saúde. Diante desse contexto, o presente artigo tem por objetivo relatar as experiências vivenciadas por seus autores nas ações de educação e cuidado em saúde promovidas pelo programa de rádio $A$ voz universitária.

\section{RELATO DE EXPERIÊNCIA}

O projeto de extensão "A voz universitária" foi concebido no contexto da pandemia da Covid-19, quando o caos se instalara em todos os lugares do mundo, incluindo o município de Altamira, no Pará. Havia uma preocupação com os povos tradicionais dessa região em virtude das dificuldades do acesso às informações de saúde, pela distância dos aldeamentos até os centros de saúde e pela ampla divulgação de informações falsas sobre a temática que chegavam até essas comunidades.

Assim, com a finalidade de levar educação em saúde no contexto da pandemia da Covid-19, sobretudo para comunidades ribeirinhas e povos tradicionais, alcançando também as comunidades rurais e urbana, a programação de A voz universitária, em parceria com a rede de Rádio e TV Vale do Xingu, é constituída por produções temáticas e semanais de podcasts com duração de um a dois minutos que vão ao ar toda quarta-feira, além de uma programação de 15 minutos apresentada todos os sábados, ao meio-dia.

O programa foi estruturado por sete discentes e um docente da Faculdade de Medicina (Famed) da Universidade Federal do Pará (Ufpa), responsáveis pela elaboração de roteiros, edição de áudios e apresentação do programa. A cada programa, foram discutidos temas variados sobre promoção da saúde, meio ambiente, bem-estar e lazer para moradores da Transamazônica e Xingu, utilizando uma linguagem compreensível e acessível.

Os programas foram organizados e produzidos em um notebook mediante auxílio de softwares. Em primeira instância, os colaboradores gravaram seus respectivos 
áudios - geralmente por diferentes aplicativos de celulares. Em segunda instância, editaram-se esses áudios no programa Audacity 2.4 a fim de corrigir ruídos, falhas e vícios vocais, bem como acrescentram-se determinados efeitos vocalizadores, como reverberação e aumento ou diminuição de amplitude e tonalidade sonora. Em terceira e última instância, utilizou-se o software Wondershare Filmora 9 com o objetivo de compilar as gravações adicionando vinhetas da mesma maneira que foram incorporadas à programação a nossa peculiaridade cultural: música regional ao fundo de toda programação, simbolizada pela guitarrada, expressivo gênero musical paraense de consumo popular, a qual é midiatizada de maneira corriqueira, assim como possui significativa expressão no espaço regional ${ }^{8}$.

O programa $A$ voz universitária, apresentado na Rádio Vale do Xingu 93.1 FM, proporcionou às comunidades citadas uma maior aproximação com a universidade. Além disso, fomentou a ampliação de conhecimentos sobre assuntos atuais e relevantes relacionados à saúde e qualidade de vida, promoveu acessibilidade às informações de maneira clara e objetiva, e contribuiu para o envolvimento da comunidade como agente ativo na promoção da própria saúde.

Executado semanalmente, o programa contou com duas fases entre os meses de julho e dezembro de 2020, totalizando 44 programas. A primeira fase teve como abordagem temas relacionados à pandemia da Covid-19, visto que, nessa época, a região do Médio Xingu passava por seu pico de transmissibilidade. Já a segunda fase, com início em outubro, teve como característica a abordagem de temáticas gerais de saúde e qualidade de vida (Tabela 1).

Tais programas alcançaram os nove municípios da região da Transamazônica, os quais fazem parte do mapa de transmissão da emissora parceira do projeto. Mais de 400 mil habitantes residem na localidade que inclui Altamira, Brasil Novo, Medicilândia, Uruará, Vitória do Xingu, Anapu, Senador José Porfírio, Porto de Moz e Placas. Ademais, devido à expressiva população ribeirinha e rural dessa região da Amazônia, as mais longínquas localidades e distritos desses municípios puderam receber, via rádio, as informações repassadas pelo programa.

Com o intuito de proporcionar informações com cunho ainda mais científico, a produção dos programas também contou com a participação de profissionais da região de diversas áreas de atuação, além de discentes de outras instituições do estado do Pará e do Brasil. Assim, foi possível gerar uma aproximação entre a comunidade ouvinte e o conhecimento técnico-científico, de modo a promover educação em saúde de maneira simples e com ampla abrangência.

Os profissionais participantes foram: enfermeiros, pediatras, ginecologistas, oncologistas, policiais rodoviários federais, biomédicos, farmacêuticos, psicólogos, educadores físicos e advogados. Além da disseminação de informações em saúde e qualidade de vida, via rádio, a plataforma das redes sociais, especialmente a do Instagram, foi utilizada para alcançar o público usuário da rede. Oito postagens informativas foram adicionadas ao perfil do projeto durante a primeira fase, além da publicação de dois podcasts na plataforma do IGTV do Instagram. De acordo com o sistema de análises de conta comercial da própria rede social, 548 contas foram alcançadas, quando se leva em conta as 11 principais publicações no perfil oficial do projeto no período de julho a dezembro.

Tabela 1. Temas abordados nos programas e podcasts.

\section{Primeira fase}

O que é a Covid-19 e como se pega?

Quais devem ser os cuidados dentro e fora de casa?

Quais materiais devem ser higienizados?

Como deve ser a higienização e por quanto tempo deve-se fazê-la?

Testes de diagnósticos para Covid-19

Quando procurar assistência em saúde e como é realizado o tratamento?

Como lidar com suspeitos/infectados em casa?

Orientações sobre a confecção e o uso de máscaras

Covid-19: Como as gestantes devem agir neste momento?

Vacinação em época de Covid-19 para crianças e adultos

Como lidar emocionalmente com a pandemia

Como cuidar das crianças em época de Covid-19

Como cuidar da saúde física em casa

\section{Segunda fase}

Prevenção do câncer de colo de útero

Planejamento familiar e prevenção de gravidez na adolescência

Prevenção ao câncer de mama

Prevenção da prematuridade e importância do pré-natal

Prevenção ao câncer de próstata

Conscientização da direção sem álcool

Prevenção de doenças sexualmente transmissíveis

Bullying na era digital

Conscientização sobre o uso de álcool e drogas

Como zelar pela saúde mental do idoso

Estratégias de prevenção a doenças cardiovasculares

Osteoporose

Uso de plantas medicinais

Fonte: Elaborado pelos autores. 
Os organizadores também elaboraram cartilhas educativas (Figura 1), em formato de gibi, visando alcançar as populações ribeirinhas que eventualmente não tivessem acesso aos demais meios de comunicação (Tabela 2). O material foi produzido de maneira artística, com o uso de elementos gráficos, com traços culturais, linguagem simples e regional, de modo que o ribeirinho criasse uma identidade com os personagens educativos.

Em parceria com o Movimento Xingu Vivo para Sempre e com a organização não governamental (ONG) Rainforest Foundation Norway, distribuíram-se aproximadamente três mil exemplares de cartilhas, uma com teor adulto e outra infantil para cada família. Elaboraram-se e veicularam-se, por meio das diferentes redes sociais, cartilhas educativas, a saber: $O$ Beiradão contra o coronavírus: orientações sobre Covid-19 para crianças ribeirinhas, Covid-19 no Beiradão: aqui você não entra não!, A turma do Zezinho contra a Covid-19, Prevenção de câncer de colo de útero no Beiradão e Educação sexual: um mundo de mudanças e descobertas.

As cartilhas foram entregues à Secretaria Municipal de Educação de Altamira objetivando maximizar seu alcance na faixa etária infantil, uma vez que o gibi pode ser repassado

Figura 1. Capa das cartilhas informativas produzidas pelo projeto.

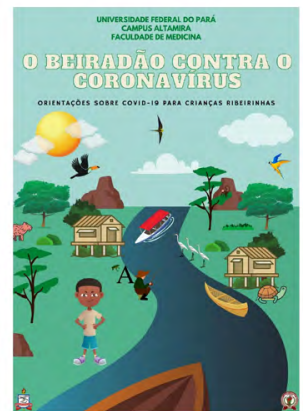

A

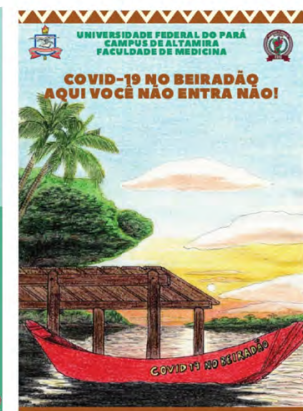

B

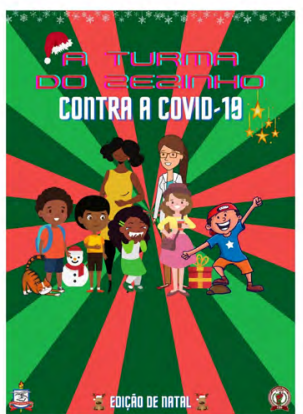

$\mathrm{C}$

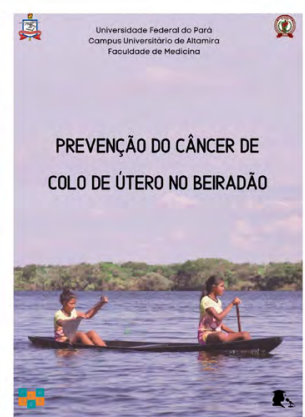

$\mathrm{D}$

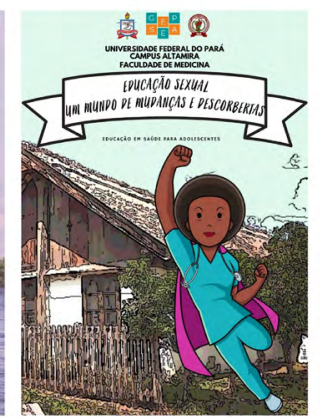

$\mathrm{E}$

(A) O Beiradão contra o coronavírus: orientações sobre Covid-19 para crianças ribeirinhas, (B) Covid-19 no Beiradão: aqui você não entra não!, (C) A turma do Zezinho contra a Covid-19, (D) Prevenção do câncer de colo de útero no Beiradão, (E) Educação sexual: um mundo de mudanças e descobertas. Fonte: Acervo do projeto.

Tabela 2. Cartilhas produzidas e distribuídas.

\section{Cartilha}

O Beiradão contra o coronavírus: orientações sobre Covid-19 para crianças ribeirinhas
Temas abordados

- Medidas preventivas contra o coronavírus.

- Importância do isolamento social.

- Grupos de risco.

- Como identificar e quando buscar ajuda médica.

- Prevenção de acidentes domésticos durante a quarentena.

Covid-19 no Beiradão: aqui você Medidas preventivas contra o coronavírus. não entra não!

- Importância do isolamento social

- Grupos de risco.

- Como identificar e quando buscar ajuda médica.

A turma do Zezinho contra a Covid-19
- Medidas preventivas contra o coronavírus.

- A importância do isolamento social.

- Grupos de risco.

- A volta às aulas no cenário da Covid-19.

- Prevenção de acidentes domésticos durante a quarentena.

\section{Prevenção do câncer de colo de útero no Beiradão
- Medidas preventi
- Fatores de risco.
Útero (PCCU).
Sinais e sintomas
- Concepção e gestação.
Educação sexual: um mundo de mudanças e descobertas
- Mudanças no corpo do adolescente.
- Métodos contraceptivos.

- Importância do Preventivo do Câncer de Colo do

Meio de divulgação

Impressão de 1.500 cópias distribuídas em comunidades ribeirinhas do Xingu, além da versão em PDF disponibilizada nas redes sociais do projeto.

Impressão de 1.500 cópias distribuídas em comunidades ribeirinhas do Xingu, além da versão em PDF disponibilizada nas redes sociais do projeto.

Versão em PDF disponibilizada à Secretaria Municipal de Educação de Altamira.

Fonte: Elaborado pelos autores. 
dos professores aos alunos durante o ensino remoto vigente, decorrente da pandemia da Covid-19.

\section{DISCUSSÃO}

As comunidades ribeirinhas da região Transamazônica e Xingu têm ficado à margem dos princípios de atenção à saúde do Sistema Único de Saúde (SUS), vivendo em situação de iniquidade pela ausência de serviços de saúde em seus diferentes níveis de complexidade. Isoladas e dispersas fisicamente, sofrem com transporte e acesso aos centros urbanos, e essa vulnerabilidade social foi potencializada pelas mudanças a partir da implantação da Usina Hidrelétrica Belo Monte, que não cumpriu as condicionantes do impacto socioambiental ${ }^{9}$.

Tais situações de fragilidade foram intensificadas com a exposição dessas comunidades à pandemia do coronavírus, pois, por conta das dificuldades de acesso às informações de saúde, das próprias barreiras geográficas e do isolamento social imposto pela pandemia, a maneira de lidar do ribeirinho com os problemas decorrentes da Covid-19 tornou-se uma incógnita. A fim de atenuarem essas fragilidades, os estudantes do curso de Medicina da Ufpa, campus de Altamira, optaram pela realização do programa de rádio $A$ voz universitária, por ser considerado um poderoso instrumento de comunicação e um importante canal para realização de iniciativas que aproximam diferentes realidades ${ }^{10}$.

Sabe-se que o rádio, apesar de toda evolução dos meios de comunicação e de suas tecnologias, ainda é o veículo que mais atinge a população brasileira, destacando-se na perspectiva de educação à comunidade, pela facilidade que as ondas eletromagnéticas do som têm em penetrar nos lugares mais distantes". Assim, a utilização da rádio local como meio para levar informações relacionadas à saúde tem se apresentado como bem-sucedida, de fácil transferibilidade e reprodução 5,12,13.

Historicamente, as transmissões de rádio foram e são utilizadas em diversos desastres naturais como método informacional. Acredita-se que, ao orientar de forma ativa a população sobre onde buscar serviços médicos e promover o aconselhamento de autocuidado, pode fazer com que os recursos médicos disponíveis sejam usados de forma mais eficaz ${ }^{6}$, além de reduzir a mortalidade em contextos não emergenciais e influenciar diretamente na transformação das atitudes de autocuidado da comunidade ${ }^{14}$.

Por essas razões, $A$ voz universitária, considerando o período correspondente e a necessidade de aliviar a sobrecarga sobre o sistema de saúde resultante da pandemia do coronavírus, concentrou-se na necessidade de sanar a carência informacional das comunidades mais distantes e também locais. Por meio de programações semanais, transmitidas em parceria com a Rádio Vale do Xingu 93.1 FM sediada no município de Altamira, foram abordados diversos assuntos de educação em saúde. Esse programa sempre priorizou o repasse de informações de forma clara e concisa, de modo a permitir a essas comunidades a possibilidade de mudança em suas condições de saúde.

A fim de que as informações fossem fixadas na mente dos ouvintes, estabeleceu-se como meta primordial a elaboração de um programa para ser veiculado aos sábados e de um programa em formato de podcast correspondente ao mesmo conteúdo, porém de maneira mais pontual e resumida, para ser transmitido diariamente. Tal método de abordagem tinha como propósito aumentar o alcance da informação e promover uma melhor fixação do assunto por meio da repetição. Esse método é eficaz para familiarizar os aprendizados e fixar conteúdos até que estes se tornem automáticos ${ }^{15}$.

Posteriormente à diminuição de casos na região, a equipe avaliou a necessidade de mudar a abordagem, e, dessa forma, elaborou-se um novo cronograma que obedecia aos meses temáticos da saúde nacional propostos pelo Ministério da Saúde. Participaram dessa ação profissionais e acadêmicos, a depender do tema. Tal estratégia, tinha como objetivo diversificar a metodologia de ensino e promover uma maior interação multidisciplinar entre profissionais e estudantes de outras áreas.

As atividades com participação desses profissionais cooperaram para a melhoria da visão holística do especialista quanto ao contexto em que a população se encontra, uma vez que muitos profissionais, quando estão inseridos em apenas um tipo de ambiente, não conhecem a realidade vivida por determinados povos social e geograficamente isolados. De acordo com Fernandez et al. ${ }^{16}$, essas populações possuem crenças e valores que as diferenciam das demais comunidades. Dessa forma, para que uma assistência em saúde seja fornecida, a fim de sanar as necessidades desse grupo, é necessário compreender essas diversidades e particularidades ${ }^{16}$.

Além disso, essas atividades propiciaram aos acadêmicos envolvidos uma experiência extensionista que perpassa os muros da universidade e que se materializou na forma de componentes extracurriculares, dando-lhes a oportunidade de desenvolver novas práticas e experiências pedagógicas no âmbito do curso de Medicina ${ }^{17}$.

Concomitantemente às programações da rádio, a equipe elaborou cartilhas elucidativas direcionadas a públicos específicos da região do Xingu. Esse material, já utilizado por Carvalho et al. ${ }^{18}$ na região, foi importante por oferecer meios para a abordagem de diferentes assuntos e realidades de forma clara e compreensível.

As duas primeiras cartilhas foram direcionadas para a comunidade ribeirinha, uma específica para o público infantil e outra para o público adulto. Ambas tinham como 
foco principal orientações sobre a Covid-19. Optou-se pela elaboração de cartilhas como instrumento infantojuvenil em razão da importância de alcançar esse público e incluí-lo no planejamento de educação em saúde, visto que esse material tinha como característica a representação do leitor por meio das histórias, de modo a promover o conhecimento e a fixação. Além disso, trata-se de uma importante ferramenta muito utilizada em campanhas governamentais como meio de aquisição de conhecimento para diferentes públicos e contextos ${ }^{19}$.

Não obstante, optou-se também pela ampla utilização de redes sociais como o Instagram e o Facebook, as quais têm se mostrado importantes veículos de disseminação capazes de ampliar a comunicação e a criação de discursos de manifestação, e abrir mais canais de transmissão de conhecimento e vinculação de educação e práticas em saúde ${ }^{20}$. Seu uso foi destinado à divulgação de informações para o público em geral por meio de banners digitais ilustrativos com textos claros, lúdicos e de fácil compreensão. As postagens ocorriam com frequência, e procurava-se a interação com o público por meio de enquetes via stories.

Ademais, foi imprescindível apontar as contribuições desse projeto para a formação médico-acadêmica e para o desenvolvimento da consciência crítica em relação a problemas de saúde nos participantes, bem como para o estímulo da busca por solução e a organização para a ação coletiva, de modo que todos passaram a ser agentes de transformação na comunidade.

Das fragilidades observadas ao decorrer do projeto, destacou-se a impossibilidade de troca de informações. Uma vez que o programa não foi realizado de forma ao vivo, não se coletou nenhuma informação instantânea do ouvinte, fragilizando de certo modo o processo educativo. Compreendese que quanto maior a participação do receptor no processo de educação, mais efetivo este é, no entanto, devido à pandemia e às medidas protetivas, o acesso à rádio tornou-se restrito, inviabilizando um programa ao vivo.

\section{CONCLUSÕES}

O papel da universidade como pilar do sistema educacional é devolver em forma de benefício à sociedade todo o investimento realizado. Nesse aspecto, o programa $A$ voz universitária teve êxito ao proporcionar, de forma simples, direta, segura e verdadeira, informações relevantes sobre saúde e qualidade de vida. Nos seus primeiros seis meses de implantação, atingiu os mais diversos públicos, desde os centros urbanos até as comunidades ribeirinhas e da zona rural da Transamazônica e Xingu por meio da rádio e de produtos educativos impressos.

Com a valorização dos profissionais da região que participaram ativamente dos programas semanais, essa ação pôde ainda reaproximar a população da academia local, além de reafirmar a importância e o valor do saber científico para o bem-estar da comunidade. Ainda, o projeto estimulou os integrantes a produzir educação em saúde por meios tecnológicos e digitais, o que gerou a consolidação dos saberes a partir da integração da comunicação e saúde, fato que assegura a democratização do acesso à saúde.

Considerando a realidade vivenciada e os aspectos culturais que permeiam as comunidades ribeirinhas alcançadas, a educação em saúde constitui um meio eficaz de promoção da saúde. Logo, atividades e projetos, como $A$ voz universitária, precisam ser constantes, visando à fortificação dos princípios de integralidade e equidade do SUS.

\section{AGRADECIMENTOS}

Os autores agradecem à Rádio Vale do Xingu 93.1 FM e também ao Ministério Público Federal a colaboração neste estudo.

\section{CONTRIBUIÇÃO DOS AUTORES}

Ana Karla da Silva Dantas participou como discente da elaboração dos roteiros, da confecção dos programas da rádio e dos podcasts, da construção das cartilhas e da escrita e formatação do manuscrito. Mirian Lourenço Santos, Lucas Mendes Carvalho, Adrianne Carla de Castro Tomé e Ramon Veloso Sousa Sobral participaram como discentes da elaboração dos roteiros, da confecção dos programas da rádio e dos podcasts, da construção de cartilhas e da escrita do manuscrito. Ozélia Sousa Santos participou como docente e supervisora das ações relatadas neste estudo pelos alunos, bem como da redação e revisão final do manuscrito.

\section{CONFLITO DE INTERESSES}

Declaramos não haver conflito de interesses.

\section{FINANCIAMENTO}

Declaramos não haver financiamento.

\section{REFERÊNCIAS}

1. Franco EC, Santo CDE, Arakawa AM, Xavier A, França MDL, Oliveira AND, et al. Promoção da saúde da população ribeirinha da região amazônica: relato de experiência. Rev. CEFAC. 2015;17(5):1521-30.

2. Ramos TM, Cabral JR, Mercês AA, Lima AF, Muniz RAA. Educação em saúde através do rádio na formação acadêmica de enfermagem. $17^{\circ} \mathrm{SENPE}$. 2013;1(1):714-6

3. James RW, Romo-Murphy E, Oczon-Quirante MM. A realist evaluation of a community-centered radio initiative for health and development in Mindanao, Philippines. Asia Pac J Public Health. 2019;31(6):559-71.

4. Torres AP, Pimenta LA, Kerbauy MTM. O uso efetivo das tecnologias de informação e comunicação (TIC) no ensino superior. Conhecimento \& Diversidade. 2018;9(18):123-43. 
5. Hugelius K, Adams M, Romo-Murphy E. The power of radio to promote health and resilience in natural disasters: a review. Int J Environ Res Public Health. 2019;16(14):2526-37.

6. Gama ASM, Fernandes TG, Parente RCP, Secoli SR. Inquérito de saúde em comunidades ribeirinhas do Amazonas, Brasil. Cad Saude Publica. 2018;34(2):e00002817.

7. Burger M. Talk radio as the soundtrack of our lives: participatory HIV/ AIDS communication, public self-expression and positive talk. SAHARA J. 2015;12(1):66-75

8. Castro FF. As guitarradas paraenses: um olhar sobre música, musicalidade e experiência cultural. As guitarradas paraenses: um olhar sobre música, musicalidade e experiência cultural//The guitarradas from Pará: A look over music, musicianship and cultural experience. Contemporânea Revista de Comunicação e Cultura. 2012;10(2):429-45.

9. Moreira RP, Herrera JA. A expansão do capital por grandes projetos: desafios ao ordenamento do território no município de Altamira-Pará. Revista Geonorte. 2013;4(12):1315-30.

10. Ramos TM. Educação em saúde através do rádio na formação acadêmica de enfermagem. Anais do 17 SENPE. 2013;1(1):714-6.

11. Santana W, Marques RRL. A comunicação radiofônica segundo moradores de uma comunidade rural em Chapada dos Guimarães-MT. Revista Comunicação, Cultura e Sociedade. 2014;4(4):1-15.

12. Da Silva BB, Travasso SQ, Mallmann DG, de Vasconcelos EMR. Uso do rádio para educação em saúde: percepção do agente comunitário em saúde. Rev Baiana Saúde Pública. 2017;41(3):734-46.

13. Roges AL, de Vasconcelos EMR, de Alencar EN, Muniz RA. Utilização do rádio pelo enfermeiro como estratégia em educação em saúde: uma revisão integrativa. Rev Eletrônica Enferm. 2013;15(1):274-81.
14. Carlos CVO, Martins EFP, Ferreira LA, Pinto MKG, Fernandes SCA. A saúde em foco na web rádio: um projeto extensionista. Revista Extendere. 2013;1(1):222-37.

15. Cocksedge KA, Guliani J, Henley W, Anderson T, Roberts S, Reed L, et al. Local radio to promote mental health awareness: a public health initiative. BJPsych Open. 2019;5(4):e60-e66.

16. Fernandez $\mathrm{AC}$, de Oliveira $\mathrm{SA}$, Lobato $\mathrm{TCL}$, Matos $\mathrm{SA}$, Xavier $\mathrm{MV}$, Albuquerque FHS, et al. Prática de campo em saúde das populações amazônicas: um relato de experiência. Revista Eletrônica Acervo Saúde. 2020;(48):e3242-e3242.

17. Krug SBF, Weigelt LD, Schultz EGR, Mocelin G, Magedanz MC, de Assis MP, et al. Projeto de educação permanente em saúde na atenção à pessoa com deficiência: contribuições no processo formativo de profissionais de saúde. Rede de cuidados à pessoa com deficiência: vivências, inovações e educação em saúde na $28^{\text {a }}$ região de sáude do Rio Grande do Sul. 23ed. 2018;01:103-12.

18. Carvalho LM, Nascimento FAA, Granato RR, Damasceno OC, Teixeira $\mathrm{FB}$, Sato DA. e-COVID Xingu: mídias sociais e informação no combate à Covid-19 em Altamira, Pará. Rev Bras Educ Med. 2020;44(sup. 1):e0142.

19. Costa AC, Gomes LMA. Elaboração de cartilha educativa para promoção do autocuidado em pacientes hipertensos e diabéticos. Trabalho de Conclusão de Curso - Universidade Federal do Piauí; 2020.

20. Murray J, Head R, Sarrassat S, Hollowell J, Remes P, Lavoie M, et al. Modelling the effect of a mass radio campaign on child mortality using facility utilisation data and the Lives Saved Tool (LiST): findings from a cluster randomised trial in Burkina Faso. BMJ Glob Health. 2018;3(4):e000808. 